\title{
Review
}

\section{Long-term harms from previous use of selective serotonin reuptake inhibitors: A systematic review}

\author{
P.B. Danborg, M. Valdersdorf and P.C. Gøtzsche* \\ Nordic Cochrane Centre, Rigshospitalet, Copenhagen, Denmark
}

\begin{abstract}
.
BACKGROUND: Millions of people are treated with antidepressants like selective serotonin reuptake inhibitors (SSRIs) or serotonin-norepinephrine reuptake inhibitors (SNRIs). This clinical practice is based on short-term trials that have exaggerated the benefits and underestimated the harms. We also know too little about long-term harms.

AIM: To assess harms of SSRIs and SNRIs that persist after end of drug intake.

METHODS: Systematic review of placebo-controlled randomised trials of any length in patients with a psychiatric diagnosis and a follow-up of at least six months. Our primary outcomes were mortality, functional outcomes, quality of life and core psychiatric events. We searched PubMed, Embase, and the Cochrane Central Register of Controlled Trials and checked the references for eligible articles. One researcher extracted data and another checked the data extraction.

RESULTS: Our searches returned 9,153 unique records. We included 22 papers for 12 trials on SSRIs. Median intervention and follow-up periods were 15 and 52 weeks, respectively. Median number of randomised participants was 51; only two trials had a drop-out rate below $20 \%$.

Outcome reporting was less thorough during follow-up than for the intervention period and only two trials maintained the blind during follow-up. All authors concluded that the drugs were not beneficial in the long term.

All trials reported harms outcomes selectively or did not report any. Only two trials reported on any of our primary outcomes (school attendance and number of heavy drinking days).

CONCLUSION: The randomised trials currently available cannot be used to investigate persistent harms of antidepressants.
\end{abstract}

Keywords: SSRI, harms, long-term, antidepressants, SNRI

\section{Background}

The number of people treated with psychiatric drugs is increasing [1-5]. Sales of selective serotonin reuptake inhibitors (SSRIs) and similar antidepressants are estimated to be so high in Denmark, that every seventh citizen can be treated with such a drug for their entire life [6].

There are many problems with placebo controlled trials of antidepressants [6-8]. The trials are of short duration, a median of only 9 weeks [8]; withdrawal effects are almost always introduced in the placebo group because the patients were already in treatment before they were randomised; lack of effective blinding; attrition; and selective reporting of major harms [6-8]. Published papers have therefore exaggerated the benefits and underestimated the harms.

${ }^{*}$ Address for correspondence: Peter C. Gøtzsche, Nordic Cochrane Centre, Rigshospitalet, Department 7811, Blegdamsvej 9, 2100 Copenhagen, Denmark. Tel.: +45 3545 7004; E-mail: pcg@ cochrane.dk. 
Despite these difficulties, we do know that short term use of antidepressants can cause irritability, anxiety and panic, emotional flattening, dyskinesias [9], sexual impairment [10,11] and also suicidality and aggression [8], even in healthy adult volunteers [12]. In observational studies, antidepressants have been linked to increased risk of dementia [13]; they may induce depressogenic effects; and the prevalence of treatment-resistant depression appears to be increasing [14]. Antidepressants have stimulant effects and rather than acknowledging this drug harm, it has led to a diagnosis of bipolar disorder in about $10 \%$ of children aged 10-14 years under the care of mental health services [15], which is a far worse disease than depression and is often treated with antipsychotics. Similar results have been reported for adults [16].

Despite the fact that we base our clinical practice on short-term trials, many patients continue on the drugs for years. A major reason for this is that they have difficulty coming off the drugs again because of withdrawal effects $[17,18]$, which for SSRIs are very similar to those the patients experience when they try to stop benzodiazepines [19].

Long-term use of psychiatric drugs may cause permanent brain damage, which has been most clearly documented for antipsychotics [20,21]. Animal studies have shown that prenatal exposure to psychiatric drugs can lead to altered physiology and behaviour later in life [22, 23].

We therefore decided to do a systematic review of long-term, or persistent, harms of treatment with SSRIs or serotonin-norepinephrine reuptake inhibitors (SNRIs) after at least six months follow-up after end of a drug treatment in humans.

\section{Methods}

\subsection{Type of studies}

We included randomised, placebo-controlled trials with a parallel group design that compared antidepressant drugs with placebo. There were no language restrictions.

\subsection{Participants}

Participants of all ages with a psychiatric diagnosis were eligible.

\subsection{Type of interventions}

Trials of any length comparing SSRIs or SNRIs as monotherapy to placebo were accepted, if the follow-up period from end of drug treatment was at least six months. We also accepted trials where at least a six-month follow-up included a tapering period.

Trials allowing concomitant medication or psychosocial interventions in both groups were accepted. Wash-out periods or placebo lead-in phases were noted. We also noted to what extent drugs are administered for side effects during follow-up, as this could potentially conceal the lasting harms of the study drugs.

\subsection{Primary outcomes}

We extracted data at the end of follow-up. Validated scales were preferred over non-validated scales. 


\subsection{Our primary outcomes}

1. Death.

2. Functional outcomes, e.g. job adherence, marital status, delinquency and traffic accidents.

3. Quality of life.

4. Core psychiatric events [24] - as listed in Appendix A.

\subsection{Secondary outcomes}

- Admission to hospital.

- Relapse/recovery rates.

- Sexual dysfunction.

- Self-rated symptom scores.

- Serious adverse events.

- Adverse events were listed under primary outcomes or serious adverse events; arbitrary thresholds for reporting were noted.

- Cognitive function, including dementia - measured on psychometric scales.

\subsection{Data collection, extraction and analysis}

Database searches were done in PubMed, Embase and the Cochrane Central Register of Controlled Trials (CENTRAL) for psychiatric drugs in general (Appendix B). For additional information on harms, we looked up the included trials in www.clinicaltrials.gov and www.clinicaltrialsregister.eu.

Screening of titles and abstracts were done by one assessor (PD). When in doubt, a second observer (PG) was involved. As our data searches were very broad (see Appendix B), we lowered the number of articles to screen by including only those abstracts that contained the word 'placebo' in Endnote, and we also excluded abstracts that contained the word 'anesthesia', as trials that tested psychiatric drugs for their anesthetic properties had inadequate drug-free follow-up periods.

Possibly eligible articles were read in full by two observers independently (PD and MV) who assessed their eligibility. One observer (PD) extracted data from the articles, which were checked by another observer (PG). Any disagreements were resolved by discussion. We reviewed the reference lists of the included papers, and if necessary, we contacted the authors of the studies to obtain more information.

We assessed the risk of bias according to the Cochrane Handbook [25] and added sponsorship bias. We also included co-morbidity bias and responder selection bias, as described in the protocol for a Cochrane review on methylphenidate in adults [26].

We performed a systematic review and had planned to perform meta-analyses but this was not possible.

\section{Results}

Our database searches identified 9153 unique records for psychiatric drugs. After screening of titles and abstracts, 262 records remained, and after full text reading we identified 40 papers with SSRIs or SNRIs as intervention drugs regardless of the indication, 22 of which were included, as they addressed participants with a psychiatric disorder. The 22 papers represented 12 unique trials (see PRISMA flow chart in Appendix B). The median publication year for the primary publication of the trials was 2013 (range 1990 to 2016).

All trials had studied SSRIs, which were fluoxetine [27-30], fluvoxamine [31-33], sertraline [34, 35], escitalopram [36, 37] and paroxetine [38] (see Table 1 for details of the trials). For three trials the 
doses were fixed $[27,32,38]$, while the remaining trials titrated the dose. The trials aimed at relieving the trial participants for symptoms of depression, post-traumatic stress disorder, obsessive-compulsive disorder, panic disorder, binge eating disorder, school refusal with anxiety and benzodiazepine use in depression. Two trials were carried out in adolescents [28, 29], while the remaining trials were in adults.

The median intervention period was 15 weeks (range 7 to 32 weeks), and the median follow-up after the randomised phase was 52 weeks (range 24 to 364 weeks) for 11 trials; the remaining trial [38] accepted varying follow-up periods ranging from four to 211 weeks). Seven trials were registered in an online trial register, and two of those had registered study results [30, 35].

The median number of participants randomized to the interventions was 51 (range 31-300). The median drop-out rate was $22 \%$ (range $10 \%$ to 52\%) among the 11 trials that provided numbers. The median drop-out rate at end of follow-up was $26 \%$ (range $12 \%$ to $50 \%$ ) among the nine trials that provided numbers. Only two trials had a drop-out rate below $20 \%[33,37]$. The median percentage of male participants was 40 (range 19 to 66) for the eleven studies with information.

The tapering method was specifically described for three trials; in one, the medication was stopped abruptly [30], and two described a four-week [31] and a six-week taper [32], respectively. The remaining trials either just mentioned a taper $[28,35,36]$, or did not mention it at all.

\subsection{Risk of bias assessments}

For sequence generation, the risk of bias was low for six trials [28, 34-38] and for allocation concealment, it was low for seven trials [28, 30, 34-38]. During the randomised phase, blinding of participants and personnel was maintained for five trials [30, 31, 34, 35, 37] and for the outcome assessors for eight trials [28, 30, 31, 33-35, 37, 38].

For blinding during the follow-up, the risk of bias amongst observers was low in two trials [30, 31], as the observers were blind to treatment conditions throughout the trial. Five trials had an unclear risk of bias, as it was not clear whether the blinding was maintained during follow-up [29, 34-37], whereas the remaining five trials had a high risk of bias because the blind was broken at the end of the intervention.

All trials reported harms outcomes selectively or did not report any. Primary outcomes as described in our methods section were only reported by two trials; one reported on school attendance [28] and the other on number of heavy drinking days [35]. For the secondary outcomes, three trials reported on remitters [27, 30, 34], and one trial reported on the self-rated Visual Analogue Scale for Depression [37].

Two trials were sponsored by both public and private grants $[32,36]$, one trial stated in the trial register that a medical centre in Israel was the sponsor but in the paper Lundbeck was the funder of the trial [37]. Two trials had no information on funding [29, 33]; the remaining trials were publicly funded. Three publicly funded trials disclosed conflicts of interest of the authors $[30,35,38]$ who were either employed in a drug company or functioned in advisory boards or as consultants for drug companies. One trial declared no conflicts of interest but was both publicly and privately funded [36]; the remaining trials shared no information on conflicts of interest.

We assessed whether co-morbidities were allowed so that the results from the clinical trials could be extrapolated to clinical practice. The risk of bias was high for all trials, as only participants with no or little co-morbidity were included. Furthermore, all trials were biased in relation to the way the participants were recruited. No trials clearly listed the participants as being treatment-naive and seven trials explicitly reported to have used either a placebo lead-in or a drug wash-out period before the randomisation [29-33, 35, 38]. Two trials did not exclude patients based on their previous responses to antidepressant drugs [28, 33]; two trials did not report on this [27, 36], while the remaining trials excluded patients based on their history or response to drugs, placebo or therapy. 


\subsection{Outcome reporting}

The reporting of harms was inadequate in all the trials. Three trials had used a scale for a systematic collection of adverse events: the 36-item somatic symptom check list [34], the Fawcett scale [32], and the 58-item side effects for children and adolescents scale [28]. For the trials that predefined the use of scales, adverse events were reported for the intervention period if there were statistically significant differences between groups and no adverse events were mentioned for the follow-up [34]; adverse events were reported if they were common (difficulty falling asleep or waking up, anger outbursts, headache, irritability, lethargy, apathy, non-suicidal self-injury ideation) [28]; or adverse events were not reported at all [32]. Two additional trials provided tables of adverse events during follow-up with no mention of choice of scale: one provided a table of serious adverse events in a trial register [35], and one provided a table of seven adverse events in the paper [37]. In summary, only three trials reported adverse events collected during follow-up [28, 35, 37] and we do not know if the reporting was complete.

The reporting of outcomes for the follow-up periods was not as thorough as the reporting for the intervention periods. One study reported that fluoxetine had a positive influence on school attendance, as ten participants $(50 \%)$ in the fluoxetine group had more than $80 \%$ school attendance at end of follow-up compared to four participants $(25 \%)$ in the placebo group [28], but several other outcomes assessed did not show statistically significant differences between groups. Based on the findings and conclusions by the authors, all trials stated that the intervention drug had not been superior to placebo, when participants were assessed at the end of follow-up, although a few among many outcomes found the tested drug to be superior [28, 33-36].

\section{Discussion}

It is well known that harms caused by SSRIs can be long-lasting [18] and there are indications that they can even be permanent, e.g. for sexual disturbances $[39,40]$. Withdrawal symptoms are also drug harms, and they can also persist for a long time [18].

Even though the median publication year was 2013, and even though all the trials we reviewed had a follow-up of at least 24 weeks after the randomised phase, none of them reported adequately on persisting harms of SSRIs; in fact, the reporting was very poor and selective. The authors were not even interested in reporting withdrawal effects although they likely occurred in all the trials.

Two trials reported on our pre-defined primary outcomes [28, 35]. As noted above, school attendance was higher for previously treated adolescents compared to placebo recipients [28], but given the number of trials and outcomes, this is likely a chance finding. For heavy drinking days there was no difference between drug and placebo at end of the 12-month follow-up [35]. For our secondary outcomes, data were sparsely reported for the follow-up periods and therefore useless. Thus, the outcomes that are relevant to the patients were almost universally ignored. Based on the reported effect outcomes, the SSRIs seemed to be no better than placebo at follow-up, also in the original authors' opinion. This is possibly an understatement, as the harms reporting was insufficient. In fact, it has been shown, based on clinical study reports obtained from the European drug regulators, that $12 \%$ more patients drop out during the randomized phase when they are on drug than when they are on placebo [41]. This suggests that placebo is a better pill than an antidepressant drug because the patients weigh the benefits against the harms when they decide whether to stay in a trial or to drop out.

A search on PubMed (31 May 2018) on "antidepressants AND placebo" limited to the article type "Randomized Controlled Trial" yielded 5,233 references. Thus, it seems that none of the authors of the thousands of placebo-controlled trials have been interested in finding out what the more lasting 
harms are of treatment with antidepressant agents. This is very worrying, particularly considering that the patients often complain about harms long after they have come off the drugs $[18,40,42]$.

Since the randomised trials are not helpful, we need to look at observational studies instead. A US national sample $(n=15,365)$ with a 9-year follow-up found that patients medicated for symptoms of major depressive disorder (measured on several parameters like the Instrumental Activities of Daily Living and the Composite International Interview short-form) had poorer long-term outcomes, based on symptom severity, than those who were not treated or used treatment other than medication [43]. Symptom severity could not be explained by covariates such as initial disease severity.

Another indication that long-term treatment with psychiatric drugs is unlikely to be beneficial is that, in all countries examined, the number of people on disability pension has gone up while usage of psychiatric drugs has also gone up [44]. By far the most used drug category is antidepressants.

Inadequate reporting of harms, even serious harms like deaths, is a general problem for psychiatric drug trials $[6,8,45,46]$, and the discrepancy between data in clinical study reports and in journal publications is disturbing $[7,12,47,48]$. This means that psychiatrists are unable to choose drug treatment strategies that optimise the benefit-to-harm balance.

Compared to the above, the co-morbidity bias we found is of minor importance. The problem with representativeness of the results from clinical trials have been highlighted before, e.g. in a study that showed that only 41 of 346 (12\%) outpatients would qualify for a clinical trial based on common inclusion and exclusion criteria for antidepressant efficacy trials [49].

Responder selection bias is also related to the representativeness of clinical trials. In eight of the included trials, there were problems related to this.

\section{Conclusion}

The currently available randomized trials are not helpful in elucidating what the persistent harms of antidepressants drugs are.

\section{Conflict of interest}

None to report.

\section{References}

[1] Kreider AR, Matone M, Bellonci C, dosReis S, Feudtner C, Huang Y-S, et al. Growth in the Concurrent Use of Antipsychotics With Other Psychotropic Medications in Medicaid-Enrolled Children. J Am Acad Child Adolesc Psychiatry. 2014;53(9):960-70.

[2] Read J, Cartwright C, Gibson K. Adverse emotional and interpersonal effects reported by 1829 New Zealanders while taking antidepressants. Psychiatry Res. 2014;216(1):67-73.

[3] Whitaker R. The anatomy of an epidemic. New York: Broadway Books; 2015.

[4] Gøtzsche PC. Deadly medicines and organised crime: How big pharma has corrupted health care. London: Radcliffe Publishing; 2013.

[5] Ilyas S, Moncrieff J. Trends in prescriptions and costs of drugs for mental disorders in England, 1998-2010. Br J Psychiatry J Ment Sci. 2012;200(5):393-8.

[6] Gøtzsche PC. Deadly psychiatry and organised denial. Copenhagen: People's Press; 2015.

[7] Maund E, Tendal B, Hróbjartsson A, Jørgensen KJ, Lundh A, Schroll J, et al. Benefits and harms in clinical trials of duloxetine for treatment of major depressive disorder: Comparison of clinical study reports, trial registries, and publications. BMJ. 2014;348:g3510. 
[8] Sharma T, Guski LS, Freund N, Gøtzsche PC. Suicidality and aggression during antidepressant treatment: Systematic review and meta-analyses based on clinical study reports. BMJ. 2016;352:i65.

[9] Goldsmith L, Moncrieff J. The psychoactive effects of antidepressants and their association with suicidality. Curr Drug Saf. 2011;6(2):115-21.

[10] Csoka AB, Csoka A, Bahrick A, Mehtonen O-P. Persistent sexual dysfunction after discontinuation of selective serotonin reuptake inhibitors. J Sex Med. 2008;5(1):227-33.

[11] La Torre A, Giupponi G, Duffy D, Conca A. Sexual dysfunction related to psychotropic drugs: A critical review-part I: Antidepressants. Pharmacopsychiatry. 2013;46(5):191-9.

[12] Bielefeldt AØ, Danborg PB, Gøtzsche PC. Precursors to suicidality and violence on antidepressants: Systematic review of trials in adult healthy volunteers. J R Soc Med. 2016;109(10):381-92.

[13] Lee CW-S, Lin C-L, Sung F-C, Liang J-A, Kao C-H. Antidepressant treatment and risk of dementia: A population-based, retrospective case-control study. J Clin Psychiatry. 2016;77(1):117-22.

[14] El-Mallakh RS, Gao Y, Jeannie Roberts R. Tardive dysphoria: The role of long term antidepressant use in inducing chronic depression. Med Hypotheses. 2011;76(6):769-73.

[15] Martin A, Young C, Leckman JF, Mukonoweshuro C, Rosenheck R, Leslie D. Age effects on antidepressant-induced manic conversion. Arch Pediatr Adolesc Med. 2004;158(8):773-80.

[16] Patel R, Reiss P, Shetty H, Broadbent M, Stewart R, McGuire P, et al. Do antidepressants increase the risk of mania and bipolar disorder in people with depression? A retrospective electronic case register cohort study. BMJ Open. 2015;5(12):e008341.

[17] Fava GA, Bernardi M, Tomba E, Rafanelli C. Effects of gradual discontinuation of selective serotonin reuptake inhibitors in panic disorder with agoraphobia. Int J Neuropsychopharmacol. 2007;10(6):835-8.

[18] Fava GA, Gatti A, Belaise C, Guidi J, Offidani E. Withdrawal Symptoms after Selective Serotonin Reuptake Inhibitor Discontinuation: A Systematic Review. Psychother Psychosom. 2015;84(2):72-81.

[19] Nielsen M, Hansen EH, Gøtzsche PC. What is the difference between dependence and withdrawal reactions? A comparison of benzodiazepines and selective serotonin re-uptake inhibitors. Addict. 2012;107(5):900-8.

[20] Ho B-C, Andreasen NC, Ziebell S, Pierson R, Magnotta V. Long-term antipsychotic treatment and brain volumes: A longitudinal study of first-episode schizophrenia. Arch Gen Psychiatry. 2011;68(2):128-37.

[21] Dorph-Petersen K-A, Pierri JN, Perel JM, Sun Z, Sampson AR, Lewis DA. The influence of chronic exposure to antipsychotic medications on brain size before and after tissue fixation: A comparison of haloperidol and olanzapine in macaque monkeys. Neuropsychopharmacol. 2005;30(9):1649-61.

[22] Oberlander TF, Gingrich JA, Ansorge MS. Sustained neurobehavioral effects of exposure to SSRI antidepressants during development: Molecular to clinical evidence. Clin Pharmacol Ther. 2009;86(6):672-7.

[23] Komatsu DE, Thanos PK, Mary MN, Janda HA, John CM, Robison L, et al. Chronic exposure to methylphenidate impairs appendicular bone quality in young rats. Bone. 2012;50(6):1214-22.

[24] Maund E, Guski LS, Gøtzsche PC. Considering benefits and harms of duloxetine for treatment of stress urinary incontinence: A meta-analysis of clinical study reports. CMAJ. 2017;189(5):E194-E203.

[25] Higgins JP, Green S. Cochrane Handbook for Systematic Reviews of Interventions [Internet]. 5.1.0. The Cochrane Collaboration; 2011. Available from: http://handbook.cochrane.org/

[26] Boesen K, Danborg PB, Gøtzsche PC, Jørgensen KJ. Extended-release methylphenidate for attention deficit hyperactivity disorder (ADHD) in adults (Protocol). Cochrane Database of Systematic Reviews 2017, Issue 11. Art. No.: CD012857.

[27] Grilo M. 12-Month Follow-up of fluoxetine and cognitive behavioral therapy for binge eating disorder. J Consult Clin Psychol. 2012;80(6):1108-13.

[28] Melvin GA, Dudley AL, Gordon MS, Klimkeit E, Gullone E, Taffe J, et al. Augmenting cognitive behavior therapy for school refusal with fluoxetine: A randomized controlled trial. Child Psychiatry Hum Dev. 2017;48(3):485-97.

[29] Simeon JG, Dinicola VF, Ferguson HB, Copping W. Adolescent depression: A placebo-controlled fluoxetine treatment study and follow-up. Prog Neuropsychopharmacol Biol Psychiatry. 1990;14(5):791-5.

[30] Vittengl JR, Clark LA, Thase ME, Jarrett RB. Predictors of longitudinal outcomes after unstable response to acute-phase cognitive therapy for major depressive disorder. Psychotherapy. 2015;52(2):268-77.

[31] Cottraux J, Mollard E, Bouvard M, Marks I. Exposure therapy, fluvoxamine, or combination treatment in obsessivecompulsive disorder: One-year followup. Psychiatry Res. 1993;49(1):63-75.

[32] de Beurs E, van Balkom AJ, Van Dyck R, Lange A. Long-term outcome of pharmacological and psychological treatment for panic disorder with agoraphobia: A 2-year naturalistic follow-up. Acta Psychiatr Scand. 1999;99(1):59-67. 
[33] Rufer M, Hand I, Alsleben H, Braatz A, Ortmann J, Katenkamp B, Fricke S, et al. Long-term course and outcome of obsessive-compulsive patients after cognitive-behavioral therapy in combination with either fluvoxamine or placebo: A 7-year follow-up of a randomized double-blind trial. Eur Arch Psychiatry Clin Neurosci. 2005;255(2):121-8.

[34] Combs K, Smith PJ, Sherwood A, Hoffman B, Carney RM, Freedland K, et al. Impact of sleep complaints and depression outcomes among participants in the standard medical intervention and long-term exercise study of exercise and pharmacotherapy for depression. J Nerv Ment Dis. 2014;202(2):167-71.

[35] Hien DA, Levin FR, Ruglass LM, López-Castro T, Papini S, Hu MC, et al. Combining seeking safety with sertraline for PTSD and alcohol use disorders: A randomized controlled trial. J Consult Clin Psychol. 2015;83(2):359-69.

[36] Kim JM, Bae KY, Stewart R, Jung BO, Kang HJ, Kim SW, et al. Escitalopram treatment for depressive disorder following acute coronary syndrome: A 24-week double-blind, placebo-controlled trial. J Clin Psychiatry. 2015;76(1):62-8.

[37] Suliman S, Seedat S, Pingo J, Sutherland T, Zohar J, Stein DJ. Escitalopram in the prevention of posttraumatic stress disorder: A pilot randomized controlled trial. BMC Psychiatry. 2015;15:24.

[38] Zitman FG, Couvée JE. Chronic benzodiazepine use in general practice patients with depression: An evaluation of controlled treatment and taper-off: Report on behalf of the Dutch Chronic Benzodiazepine Working Group. Br J Psychiatry. 2001;178:317-24.

[39] Healy D, Le Noury J, Mangin D. Enduring sexual dysfunction after treatment with antidepressants, $5 \alpha$-reductase inhibitors and isotretinoin: 300 cases. Int J Risk Saf Med. 2018;29(3-4):125-34.

[40] Danborg PB, Simonsen AL, Hróbjartsson A, Gøtzsche PC. Long-term changes in observed behavior after exposure to psychiatric drugs: A systematic review of animal studies. Published online, http://nordic.cochrane.org/sites/ nordic.cochrane.org/files/public/uploads/danborg_2017_long-term_harms_animal_study.pdf

[41] Sharma T. Effects of selective serotonin reuptake inhibitors (SSRIs) and serotonin - norepinephrine reuptake inhibitors (SNRIs) on suicidality, violence, and quality of life ( $\mathrm{PhD}$ Thesis): University of Copenhagen; 2018.

[42] Coming off antidepressants - a survey by the Royal College of Psychiatrists. 2014. Was available from: http://www.rcpsych.ac.uk/healthadvice/treatmentswellbeing/antidepressants/comingoffantidepressants.aspx (accessed February 3, 2016).

[43] Vittengl JR. Poorer long-term outcomes among persons with major depressive disorder treated with medication. Psychother Psychosom. 2017;86(5):302-4.

[44] Whitaker R. Anatomy of an Epidemic. New York: Broadway Paperbacks; 2015.

[45] Jakobsen JC, Katakam KK, Schou A, Hellmuth SG, Stallknecht SE, Leth-Møller K, et al. Selective serotonin reuptake inhibitors versus placebo in patients with major depressive disorder. A systematic review with meta-analysis and Trial Sequential Analysis. BMC Psychiatry. 2017;17(1):58.

[46] Hughes S, Cohen D, Johnson R. Adverse event assessment methods in published trials of psychotropic drugs: Poor reporting and neglect of emerging safety concerns. Int J Risk Saf Med. 2016;28(2):101-14.

[47] Roest AM, de Jonge P, Williams CD, de Vries YA, Schoevers RA, Turner EH. Reporting Bias in Clinical Trials Investigating the Efficacy of Second-Generation Antidepressants in the Treatment of Anxiety Disorders: A Report of 2 Meta-analyses. JAMA Psychiatry. 2015;72(5):500-10.

[48] Golder S, Loke YK, Wright K, Norman G. Reporting of adverse events in published and unpublished studies of health care interventions: A systematic review. PLoS Med. 2016;13(9):e1002127.

[49] Zimmerman M, Mattia JI, Posternak MA. Are subjects in pharmacological treatment trials of depression representative of patients in routine clinical practice? Am J Psychiatry. 2002;159(3):469-73.

[50] Blumenthal JA, Babyak MA, Doraiswamy PM, Watkins L, Hoffman BM, Barbour KA, et al. Exercise and pharmacotherapy in the treatment of major depressive disorder. Psychosom Med. 2007;69(7):587-96.

[51] Hoffman BM, Babyak MA, Craighead WE, Sherwood A, Doraiswamy PM, Coons MJ, et al. Exercise and pharmacotherapy in patients with major depression: One-year follow-up of the SMILE study. Psychosom Med. 2011;73(2):127-33.

[52] Cottraux J, Mollard E, Bouvard M, Marks I, Sluys M, Nury AM, et al. A controlled study of fluvoxamine and exposure in obsessive-compulsive disorder. Int Clin Psychopharmacol. 1990;5(1):17-30.

[53] de Beurs E, van Balkom AJ, Lange A, Koele P, van Dyck R. Treatment of panic disorder with agoraphobia: Comparison of fluvoxamine, placebo, and psychological panic management combined with exposure and of exposure in vivo alone. Am J Psychiatry. 1995;152(5):683-91.

[54] Kim JM, Bae KY, Kang HJ, Kim SW, Shin IS, Hong YJ, et al. Design and Methodology for the Korean Observational and Escitalopram Treatment Studies of Depression in Acute Coronary Syndrome: K-DEPACS and EsDEPACS. Psychiatry Investig. 2014;11(1):89-94.

[55] Kim JM, Stewart R, Bae KY, Kang HJ, Kim SW, Shin IS, et al. Correlates and Escitalopram Treatment Effects on Sleep Disturbance in Patients with Acute Coronary Syndrome: K-DEPACS and EsDEPACS. Sleep. 2015;38(7):1105-11. 
[56] Kim SY, Stewart R, Bae KY, Kim SW, Shin IS, Hong YJ, et al. Influences of the Big Five personality traits on the treatment response and longitudinal course of depression in patients with acute coronary syndrome: A Randomised controlled trial. J Affect Disord. 2016;203:38-45.

[57] Tonge BJ, Melvin GA, Dudley AL, Gordon MS. Cognitive behavioural therapy with or without fluoxetine for school refusal: A Randomised controlled trial. RANZCP. 2013;47:59, abstract for Royal Australian and New Zealand College of Psychiatrists, RANZCP Annual Congress 2013 Sydney Australia.

[58] Vittengl JR, Clark LA, Thase ME, Jarrett RB. Stable remission and recovery after acute-phase cognitive therapy for recurrent major depressive disorder. J Consult Clin Psychol. 2014;82(6):1049-59.

[59] Jarrett RB, Thase ME. Comparative efficacy and durability of continuation phase cognitive therapy for preventing recurrent depression: Design of a double-blinded, fluoxetine- and pill placebo-controlled, randomized trial with 2-year follow-up. Contemp Clin Trials. 2010;31(4):355-77.

[60] Jarrett RB, Minhajuddin A, Gershenfeld H, Friedman ES, Thase ME. Preventing depressive relapse and recurrence in higher-risk cognitive therapy responders: A Randomized trial of continuation phase cognitive therapy, fluoxetine, or matched pill placebo. JAMA Psychiatry. 2013;70(11):1152-60.

\section{Appendix A - overview of psychiatric adverse events, as used in Bielefeldt et al., and Maund et al.}

List of terms for suicidality, violence, and proposed driving mechanisms for these events Table of terms were developed based on:

- FDA clinical review of the relationship between antidepressants and suicidality in adults

- Literature Review - Sinclair et al., Price et al. and reference checking

- Email and teleconference with Professor David Healy

\begin{tabular}{ll}
\hline Suicidality & DH comments \\
\hline Core event & \\
Accident & \\
Attempt & \\
Burn & \\
Cut & \\
Drown & \\
Gas \\
Gun \\
Hang \\
Hung \\
Immolat \\
Injur \\
Jump \\
Monoxide \\
Mutil \\
Overdose \\
Salf damage \\
Self harm \\
Self inflicted \\
Self injury \\
Shoot \\
Suici \\
\hline
\end{tabular}


(Continued)

\begin{tabular}{l}
\hline Suicidality $\quad$ DH comments \\
\hline Poison \\
Asphyxiation \\
Suffocation \\
Firearm
\end{tabular}

\begin{tabular}{lll}
\hline Violence & Synonyms & DH comments \\
\hline
\end{tabular}

\section{Core event}

Homicide

Physical assault

Physical abuse

Homicidal ideation

Violence-related symptom
Assaultive behaviour

Criminal behaviour,

Antisocial behaviour,

Physically threatening behaviour

\begin{tabular}{ll}
\hline Activation event & Synonyms \\
\hline $\begin{array}{l}\text { Core event } \\
\text { Jitteriness }\end{array}$ & Jittery \\
Shakiness & Inner shakiness \\
Irritability & Increased irritability, hostility, frustration \\
Increased energy & Increased energy, excessive energy, \\
& unusual energy, feeling euphoric, \\
& euphoria, CNS stimulation, \\
& overstimulation, caffeine feeling, \\
& amphetamine speed like response, racing \\
& thoughts
\end{tabular}

Mania (also listed under psychotic event)

Anxiety

Agitation

Restless

Akathisia

\section{Potential event}

Insomnia

Tremor

Panic

Tension

Unable to relax

Easily startled

Panic attack, panic symptoms

Tension increased, tenseness

ncreased anxiety, overanxious, nervousness, anxiety attack

Increased agitation, aggression, hostility, argumentative

Restlessness, motor restlessness, fidgetiness, hyperactivity, hyperkinesia, "skin crawling"
Should be included as core event

Should be included as core event

Drs may use mania for both activation and psychotic events. Patients report being "manic" when they mean more active than normal i.e. experiencing activation. $\begin{array}{ll}\text { Insomnia, sleeplessness, } & \text { Not really an activation symptom } \\ \text { Trembling } & \text { Should be a potential event }\end{array}$

$\begin{array}{ll}\text { Insomnia, sleeplessness, } & \text { Not really an activation symp } \\ \text { Trembling } & \text { Should be a potential event }\end{array}$

DH comments 


\begin{tabular}{|c|c|c|}
\hline Psychotic event & Synonyms & DH comments \\
\hline \multicolumn{3}{|l|}{ Core event } \\
\hline \multicolumn{3}{|l|}{ Hallucinations } \\
\hline \multicolumn{3}{|l|}{ Paranoia } \\
\hline \multicolumn{3}{|l|}{ Feelings of doom } \\
\hline \multicolumn{3}{|l|}{ Delusions } \\
\hline \multicolumn{3}{|l|}{ Delirium } \\
\hline \multicolumn{3}{|l|}{ Psychosis } \\
\hline Abnormal thinking & $\begin{array}{l}\text { Intrusive thoughts, unusual thoughts, } \\
\text { abnormal feelings }\end{array}$ & \\
\hline Confusion & Disorientation, incoherent thoughts & $\begin{array}{l}\text { Comment EM: "spaciness" was a verbatim } \\
\text { term being coded as confusion in tables for } \\
\text { SER trials but DH said verbatim term of } \\
\text { "spaciness" should be considered emotional } \\
\text { disturbance }\end{array}$ \\
\hline \multicolumn{3}{|l|}{ Behavioural dyscontrol } \\
\hline $\begin{array}{l}\text { Manic reaction (also listed } \\
\text { under activation) }\end{array}$ & Hypomania, euphoria & \\
\hline \multicolumn{3}{|l|}{ Hysteria } \\
\hline \multicolumn{3}{|l|}{ Potential event } \\
\hline Nightmares & Bad dreams & \\
\hline Abnormal dreams & $\begin{array}{l}\text { Strange dreams, increased dreams, vivid } \\
\text { dreams, intense dreams }\end{array}$ & \\
\hline
\end{tabular}

\section{Core event}

Flat effect

Apathy

Anhedonic

Disinhibition

Derealisation

Depersonalisation

Indifference
Depersonalised

Flattened, reduced ability to feel positive

emotions, feeling blank

Emotionally detached

Lack of empathy

Impulsivity

Emotional lability

Spaciness
This is not an especially psychotic event and belong with emotional disturbance 


\section{Appendix B - flow diagram and information on searches}

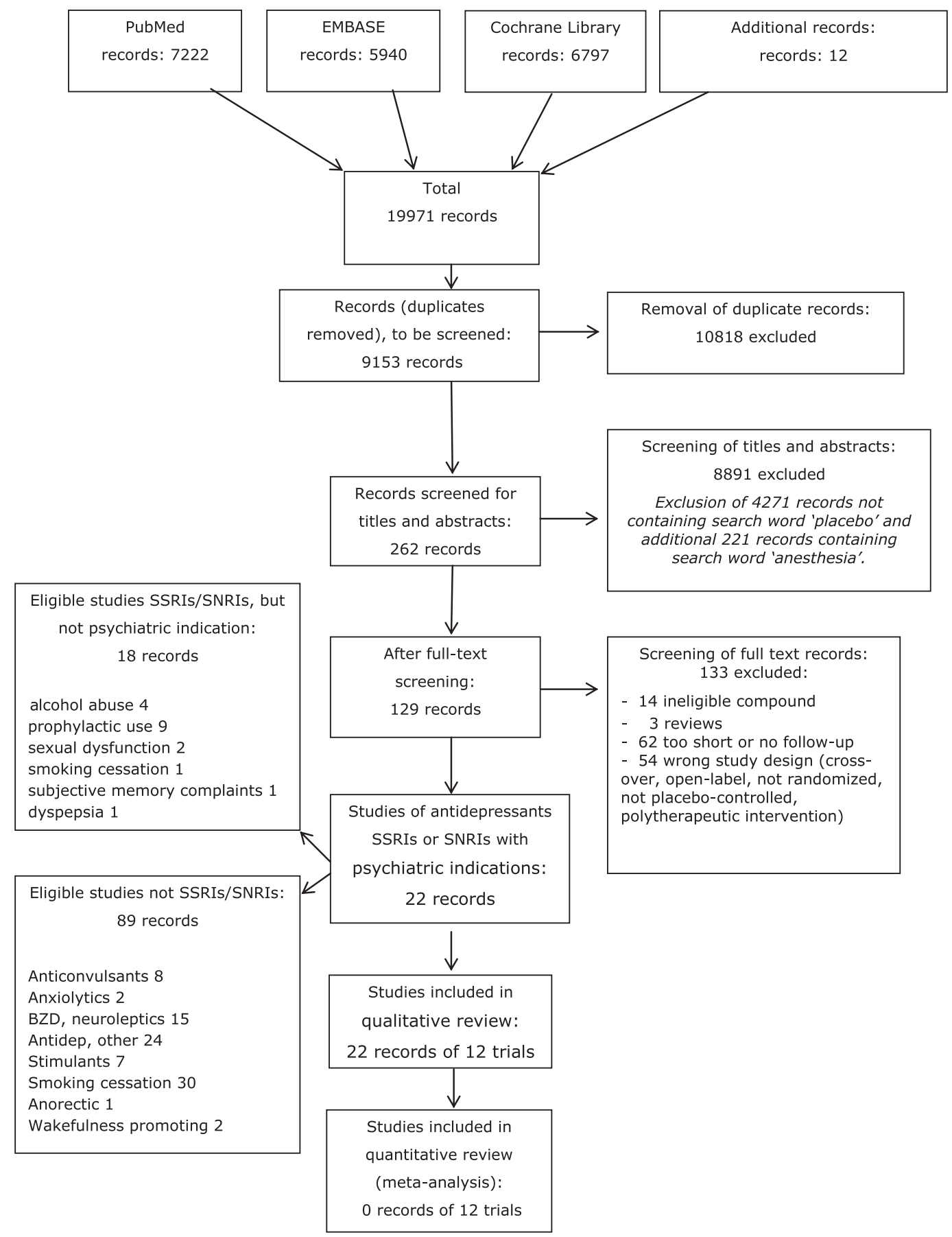




\section{Information on searches}

For PubMed and Embase:

Filters applied: 'Randomized Controlled Trial' and 'Humans'

For indexed MeSH/Map terms, we will restrict to Major Topic (PubMed) and Focus (Embase) to include studies with main focus on the selected topic for the drug categories.

\section{All databases:}

Narrowing down to trials with follow-up we will apply the following terms: "Follow-Up Studies"[MeSH], discontinuation[tiab], maintenance[tiab], follow-up[tiab], long-term[tiab], withdrawal[tiab], extension[tiab], substance withdrawal syndrome[MeSH].

[tiab] searches in PubMed are equivalent to.mp searches in Embase and :ti,ab searches in CENTRAL. Equivalent searches were applied in the three databases.

Example, PubMed search categories, MeSH:

"Anti-Anxiety Agents" [Pharmacological Action]

"Anticonvulsants" [Pharmacological Action]

"Barbiturates"[Mesh]

"Serotonin Uptake Inhibitors" [Pharmacological Action]

"Antidepressive Agents" [Pharmacological Action]

"Monoamine Oxidase Inhibitors"[Mesh]

"Benzodiazepines"[Mesh]

"Antipsychotic Agents" [Pharmacological Action]

"Guanfacine"[MeSH]

"Clonidine”[Mesh]

"Hypnotics and Sedatives" [Pharmacological Action]

"Propylamines"[Mesh] incl atomoxetine and fluoxetine

"Central Nervous System Stimulants" [Pharmacological Action] incl. methylphenidate and guanfacine, amphetamine, lisdexamphetamine, pemoline, modafinil (tested but rejected for ADHD)

"Tranquilizing Agents"[Mesh]

+text words [tiab/.mp/:ti,ab]: SSRI, antidepressant, methylphenidate, anticonvulsant, benzodiazepine, antipsychotics

For Embase search: explode-function and include all subheadings-function was applied to include subgroups. 